\title{
Oficinas Terapêuticas: Relato de Experiência sobre os Instrumentos de Cuidado Relacionados ao Empreendedorismo em um CAPS no Estado do Pará
}

\author{
Therapeutic Workshops: Experience Report on the Care Instruments Related to \\ Entrepreneurship in a CAPS in the State of Pará
}

Talleres Terapéuticos: Relato de Experiencia sobre los Instrumentos de Cuidado Relacionados al Espíritu Empresarial en un CAPS en el Estado de Pará

Maiza Silva de Sousa ${ }^{1 *}$, Ana Beatriz Sousa Alves ${ }^{1}$, Juliana de Fátima Almeida da Penha ${ }^{1}$, Karen Alessandra de Jesus Cuimar ${ }^{1}$, Márcio Yrochy Saldanha dos Santos ${ }^{1}$, Karollyne Quaresma Mourão ${ }^{1}$.

\section{RESUMO}

Objetivo: Relacionar o empreendedorismo social às oficinas terapêuticas a partir de uma experiência de acadêmicos de enfermagem em um Centro de Atenção Psicossocial (CAPS). Métodos: Trata-se de estudo descritivo, exploratório, observacional, o qual seguiu a metodologia da problematização do Arco de Maguerez. A pesquisa foi realizada em um CAPS modalidade III, situado em um bairro de classe média em Belém, Pará. Resultados e Discussões: As oficinas terapêuticas funcionam como instrumento de cuidado em saúde mental, nas quais são fortalecidos os vínculos entre os usuários e profissionais, bem como são valorizadas e respeitadas às individualidades de cada um, dando espaço para expressão e exteriorização de pensamentos e sentimentos, colaborando com seu projeto terapêutico singular e sua reinserção social. Diante da falta de recursos para manter as oficinas terapêuticas realizadas no CAPS, foi proposta a criação de uma loja virtual cujo objetivo voltava-se à venda dos produtos customizados pelos próprios usuários, e consequentemente arrecadar fundos para dar continuidade a esse recurso terapêutico. Conclusão: A implantação do empreendedorismo no CAPS, através da loja virtual, pode propiciar o incentivo à execução das oficinas terapêuticas e dar continuidade a outras atividades desenvolvidas no CAPS.

Palavras-chave: Saúde Mental, Enfermagem, Empreendedorismo, Tecnologia.

\begin{abstract}
Objective: To relate social entrepreneurship to therapeutic workshops based on an experience of nursing academics in a Psychosocial Care Center (CAPS). Methods: This is a descriptive, exploratory, observational study, which followed the methodology of the problematization of the Arch of Maguerez. The research was carried out in a modality III CAPS, located in a middle-class neighborhood in Belém, Pará. Results and Discussions: Therapeutic workshops function as an instrument of mental health care, in which the links between users and professionals are strengthened, as well as are valued and respected to the individualities of each one, giving space for expression and externalization of thoughts and feelings, collaborating with its unique therapeutic project and its social reintegration. Given the lack of resources to maintain the therapeutic workshops held at the CAPS, it was proposed to create a virtual store whose objective was to sell customized products by the users themselves, and consequently raise funds to continue this therapeutic resource. Conclusion: The implementation of entrepreneurship in the CAPS, through the virtual store, can encourage the execution of therapeutic workshops and give continuity to other activities developed in the CAPS.
\end{abstract}

Keywords: Mental Health, Nursing, Entrepreneurship, Technology.

1Universidade do Estado do Pará (UEPA), Belém - Pará. *E-mail: maizasousa1619@gmail.com

SUBMETIDO EM: 4/2019

ACEITO EM: 5/2019

PUBLICADO EM: 7/2019

REAS/EJCH | Vol.Sup.25 | e731 | DOI: https://doi.org/10.25248/reas.e731.2019 Página 1 de 7 


\section{RESUMEN}

Objetivo: Relacionar el empredimiento social a los talleres terapéuticos a partir de una experiencia de académicos de enfermería en un Centro de Atención Psicosocial (CAPS). Métodos: Se trata de estudio descriptivo, exploratorio, observacional, el cual siguió la metodología de la problematización del Arco de Maguerez. La investigación fue realizada en un CAPS modalidad III, que está ubicado en un barrio de clase media en Belém, Pará. Resultados y Discusiones: Los talleres terapéuticos funcionan como instrumento de cuidado en salud mental, en los cuales son fortalecidos los vínculos entre los usuarios y profesionales, bien como son valoradas y respetadas las individualidades de cada uno, dando espacio para expresión y exteriorización de pensamientos y sentimientos, colaborando con su proyecto terapéutico singular y su reinserción social. Frente la falta de recursos para mantener los talleres terapéuticos realizados en CAPS, fue propuesta la creación de una tienda virtual cuyo objetivo se enfocaba en la venta de los productos personalizados por los propios usuarios, y, consecuentemente, recaudar fondos para dar continuidad a este recurso terapéutico. Conclusión: La implantación del espíritu empresarial en el CAPS, a través de la tienda virtual, puede propiciar el incentivo a la ejecución de los talleres terapéuticos y dar continuidad a otras actividades desarrolladas en el CAPS.

Palabras clave: Salud Mental, Enfermería, Espíritu Empresarial, Tecnología.

\section{INTRODUÇÃO}

O Centro de Atenção Psicossocial (CAPS) surgiu no Brasil, em São Paulo, no ano de 1987. Mantido com recursos federais e de caráter público, esse serviço novo veio suprir a demanda de atendimentos em saúde mental, sendo chamado primeiramente de Centro de Atenção Psicossocial Luiz da Rocha Cerqueira. Posteriormente, a sigla CAPS foi padronizada em todo o país (PITTA A, 1996).

Os CAPS são centros estratégicos que integram a Rede de Atenção Psicossocial (RAPS), oferecendo serviços de saúde de caráter aberto e comunitário, através de uma equipe multiprofissional que realiza atendimentos às pessoas com sofrimento mental, incluindo dependentes químicos, em situação de crise ou no processo de reabilitação psicossocial, onde se faz uso de diversas estratégias terapêuticas (BRASIL, 2004).

Entre essas estratégias, destaca-se a oficina terapêutica que, segundo Ribeiro LA, et al., (2008) são métodos operacionais de reabilitação psicossocial, na qual se promove a socialização, o acolhimento, a expressão e o desenvolvimento de habilidades motoras, através da produção dos mais diversos materiais, como pinturas, desenhos e demais artesanatos, que podem vir a ser comercializados, configurando uma modalidade de empreendedorismo dentro do CAPS.

Os CAPS são divididos em sete modalidades: CAPS I, CAPS II, CAPS i, CAPS AD (Álcool e Drogas), CAPS III, CAPS ad III (Álcool e Drogas) e CAPS ad IV. Tais modalidades atendem pessoas em sofrimento mental e/ou problemas decorrentes do uso de álcool e outras drogas, diferenciando-se pelo público alvo atendido, faixa etária e o contingente populacional da região onde o CAPS está inserido (BRASIL, 2017).

Segundo Mason C, et al., (2015) o empreendedorismo é caracterizado como práticas inovadoras que podem ser utilizadas para o benefício de uma comunidade, o que ele chama de empreendedorismo social. Neste caso, na área da saúde o empreendedorismo social pode ser utilizado a fim de proporcionar a equidade dos cuidados em saúde no CAPS. Atrelado às perspectivas inovadoras estão às mídias digitais, importantes ferramentas de divulgação, interação e comercialização dos mais diversos produtos que podem ser utilizadas ao benefício comunitário (PEREIRA JA e BERNARDO A, 2016; NUNES BS, et al., 2018). Gandolfi FA, et al.,(2017) nomeou algumas mídias digitais como Tecnologias Interativas da Web (TIW) e segundo ele constituem-se em espaços virtuais que podem proporcionar ampla funcionalidade e praticidade para as pessoas, pois permitem a troca de informações, a interação e comunicação interpessoal por meio de mensagens instantâneas, a propagação de notícias, além de ambiente para estabelecimento de novas formas de trabalho. 
A criação de perfis comerciais nas mídias digitais, como o Instagram ${ }^{\circledR}$ e Facebook ${ }^{\circledR}$, permite apropriar-se de um método cada vez mais comum para empreender um novo negócio, pois garante amplo alcance do público e, com isso, maior facilidade na obtenção de retorno financeiro (JÚNIOR VEM, 2018).

Assim, este estudo tem como proposta a criação de uma loja virtual, a fim de divulgar os produtos confeccionados pelos usuários do CAPS durante as oficinas terapêuticas, de modo que proporcione a obtenção de renda, que poderá ser revestida em benefício da instituição como um todo, com foco em manter o funcionamento de tais oficinas.

\section{MÉTODOS}

Trata-se de um estudo descritivo, exploratório, do tipo observacional, desenvolvido durante as aulas práticas do componente curricular Enfermagem em Atenção à Saúde Mental I, do Curso de Graduação em Enfermagem da Universidade do Estado do Pará (UEPA). Nas quais se pretendeu abordar a dinâmica de um CAPS sob a perspectiva do empreendedorismo na enfermagem, seguindo a Metodologia da Problematização do Arco de Maguerez, que se divide em cinco etapas.

De acordo com Berbel NAN, (2011), as etapas da Metodologia da Problematização são: (1) Observação da realidade, em que os pesquisadores são levados a analisar os aspectos sociais e concretos do ambiente a ser estudado; (2) Levantamento dos pontos chave, que consiste em destacar os problemas e refletir acerca deles, o que irá direcionar o próximo passo; a partir dessas duas etapas tem-se definido o que será trabalhado.

A etapa seguinte é a (3) Teorização, a qual é baseada no levantamento bibliográfico das questões anteriormente encontradas, a fim de encontrar subsídios para desenvolver a etapa seguinte; (4) Hipóteses de solução, na qual se buscam maneiras de resolver o problema; e (5) Aplicação à realidade, momento no qual os pesquisadores voltam a campo e colocam em prática as medidas de resolução do problema encontrado (BERBEL NAN, 2011). O local escolhido para a realização do estudo foi um CAPS modalidade III, localizado em um bairro de classe média entre a periferia e o centro da cidade de Belém, Pará. Nesse ambiente são realizadas semanalmente 16 oficinas terapêuticas que atendem em média 550 usuários.

De acordo com a primeira e segunda etapa proposta no Arco de Maguerez, na primeira visita ao local, identificou-se que um dos maiores problemas do CAPS era a carência de recursos financeiros e de materiais para a manutenção das oficinas terapêuticas. Sendo assim, os pesquisadores decidiram aplicar o empreendedorismo através da construção de uma loja virtual para vender os produtos confeccionados nas oficinas, visando obter lucro para mantê-las.

Seguindo a metodologia, na etapa de Teorização optou-se por trabalhar duas categorias: I. Oficina Terapêutica no CAPS e II. Empreendedorismo como Estratégia de Cuidado, que surgiram a partir dos problemas encontrados na realidade local e da investigação na literatura. Como hipótese de solução foi proposta a construção de uma loja virtual e o desenvolvimento de uma oficina terapêutica com os usuários. No retorno à realidade foi entregue o projeto da loja virtual ao coordenador do CAPS e realizada uma oficina terapêutica com 40 usuários do serviço, através da qual foram construídos brindes, que deveriam ser entregues a uma pessoa considerada especial ao usuário.

\section{RESULTADOS e DISCUSSÕES}

A partir da observação da realidade e da teorização, os resultados encontrados e suas respectivas discussões foram divididos em duas categorias: Oficina Terapêutica no CAPS; e Empreendedorismo como Estratégia de Cuidado.

\section{Oficina Terapêutica no CAPS}

O CAPS é um serviço de saúde mental que visa substituir e descentralizar o hospital psiquiátrico, gerando um novo modelo de atenção à saúde mental (IBIAPINA ARS, et al., 2017). Além disso, no CAPS se 
disponibiliza assistência e reabilitação psicossocial, dando acesso a diversos aspectos da vida, como trabalho, cultura, lazer e educação, fortalecendo os laços do usuário com a família e comunidade (RIBEIRO LA, et al.,2008). Partindo desse princípio, foi proposto, como retorno a realidade, a construção e a aplicação de uma oficina terapêutica, conciliando o desenvolvimento das habilidades psicomotoras ao empreendedorismo social. Além disso, a oficina teve um significado alusivo ao Dia Mundial da Saúde Mental, incentivando o autocuidado e a valorização dos usuários.

Segundo Ribeiro LA, et al. (2008) as oficinas terapêuticas são estratégias de tratamento, cuidado e socialização, que constituem uma maneira de acolhimento, convivência, interação e diálogo. Ademais, funcionam como organizador do cuidado em saúde mental, na qual se produzem vínculos entre os usuários e se valorizam as individualidades de cada um, dando espaço para expressão e exteriorização de pensamentos e sentimentos, colaborando com seu projeto terapêutico singular e sua reinserção social (IBIAPINA ARS, et al., 2017).

Tendo isso em vista, a oficina proposta na ação foi a construção de uma cestinha para presente. Esta foi desenvolvida com o uso de Ethylene Vinyl Acetate (E.V.A) previamente cortados nos formatos necessários para a cesta, cola de isopor, fita colorida, saco plástico pequeno e bombons de chocolate. Iniciou-se com a explicação de como deveria ser montada a cesta; posteriormente foram distribuídos os kits para a confecção da mesma, cabendo aos pesquisadores à supervisão e auxílio aos usuários durante a montagem da cesta. Um modelo da cesta construída durante a ação pode ser observado na Figura 1 abaixo.

Figura 1 - Modelo de cesta produzida na oficina. Belém-PA, 2018.

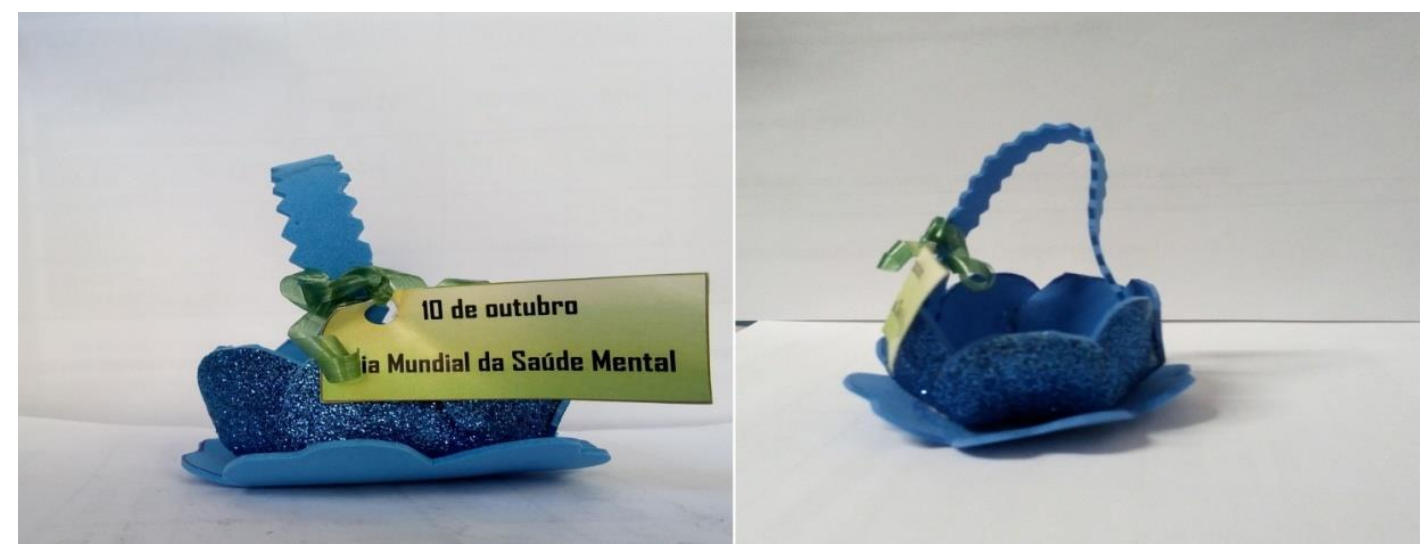

Fonte: Arquivo de SOUSA MS, 2019.

Deste modo, a oficina aplicada durante a ação, proporcionou aos usuários o uso de habilidades psicomotoras para a montagem da cestinha, além de estimular seus aspectos afetivos e emocionais, pois foi sugerido que a cestinha, depois de finalizada, poderia ser entregue a uma pessoa especial, como um membro da família ou um amigo, fortalecendo e incentivando o vínculo do usuário com estas pessoas, que também têm importância significativa no tratamento deste. Além disso, as oficinas terapêuticas constituem-se como um dos principais métodos de tratamento ofertados nos CAPS.

Visam estimular a integração social e familiar, a manifestação de sentimentos e problemas, o desenvolvimento de habilidades corporais, realização de atividades produtivas e exercício da cidadania. Dependendo da finalidade a qual se direcionam, podem ser de diferentes tipos, como oficinas expressivas, oficinas de alfabetização e oficinas geradoras de renda (BRASIL, 2004).

Sabe-se que desde a Reforma Psiquiátrica há a preocupação de proporcionar aos portadores de sofrimento psíquico uma reinserção social. Essa mudança de olhar teve início com a segunda metade do século $X X$, onde surgiram os fármacos, que amenizam os sintomas e possibilitam certo nível de retorno à 
consciência, proporcionando assim um tratamento humanizado em serviços de saúde, que possibilitem o cuidado e a produção de novas relações (TENÓRIO F, 2002).

Uma das características marcantes desse novo serviço proposto é a variedade de terapêuticas empregadas, principalmente nos CAPS, que tem como objetivo, segundo o Ministério da Saúde, reintegrar socialmente os pacientes através do uso das oficinas terapêuticas, que podem dar acesso ao mercado de trabalho, lazer, fortalecimento dos laços familiares e comunitários (BRASIL, 2004). Assim, as oficinas terapêuticas buscam incentivar a reinserção social, através da troca de conhecimentos, bem como proporcionar ao usuário autonomia de direito, visto que as oficinas desenvolvem suas noções e funções psicomotoras, e ajudam nas tomadas de decisão, o que é muito importante na reinserção social.

Segundo Ribeiro LA, et al., (2008), outras finalidades das oficinas terapêuticas, além de tratamento e interação social, é a geração de renda para manutenção das instituições. Sendo assim, as oficinas podem ser utilizadas como formas de empreender dentro dos CAPS, uma vez que os produtos confeccionados nessas atividades têm potencial para se tornarem itens comerciais.

\section{Empreendedorismo como Estratégia de Cuidado}

O termo empreendedorismo é de origem bastante antiga e segundo Gouveia IM e Pinto JP (2013), deriva do francês entreprendre, que significa "fazer algo" ou "empreender"; entre é oriundo do latim inter e quer dizer ação mútua, reciprocidade e pendre do latim prehendere, que significa utilizar, tomar uma atitude. Sendo assim, empreendedorismo é definido como a inovação ou aperfeiçoamento de algo, visando beneficiar os indivíduos e a sociedade (ANDRADE AC, et al., 2015).

Atualmente muito tem se falado das práticas inovadoras na área da saúde, o que se conhece como empreendedorismo social. Mason C, et al., (2015) afirma que essas medidas constituem ferramenta útil para proporcionar a equidade em saúde, uma vez que a utilização de novos recursos sociais, culturais e materiais contribuem para a mobilização do usuário, valorizando seus conhecimentos e experiências individuais, o que assegura a promoção a saúde e melhoria da qualidade de vida.

Diante da falta de recursos para manter as oficinas terapêuticas realizadas no CAPS em que se realizou a pesquisa, foi proposta a criação de uma loja virtual cujo objetivo seria vender os produtos customizados pelos próprios usuários e arrecadar fundos para dar continuidade a esse recurso terapêutico. Um modelo de loja virtual proposta para o CAPS com endereço eletrônico no Instagram está identificado na Figura 2 abaixo.

Figura 2 - Loja virtual criada para vender os produtos customizados no CAPS. Belém PA, 2018. 


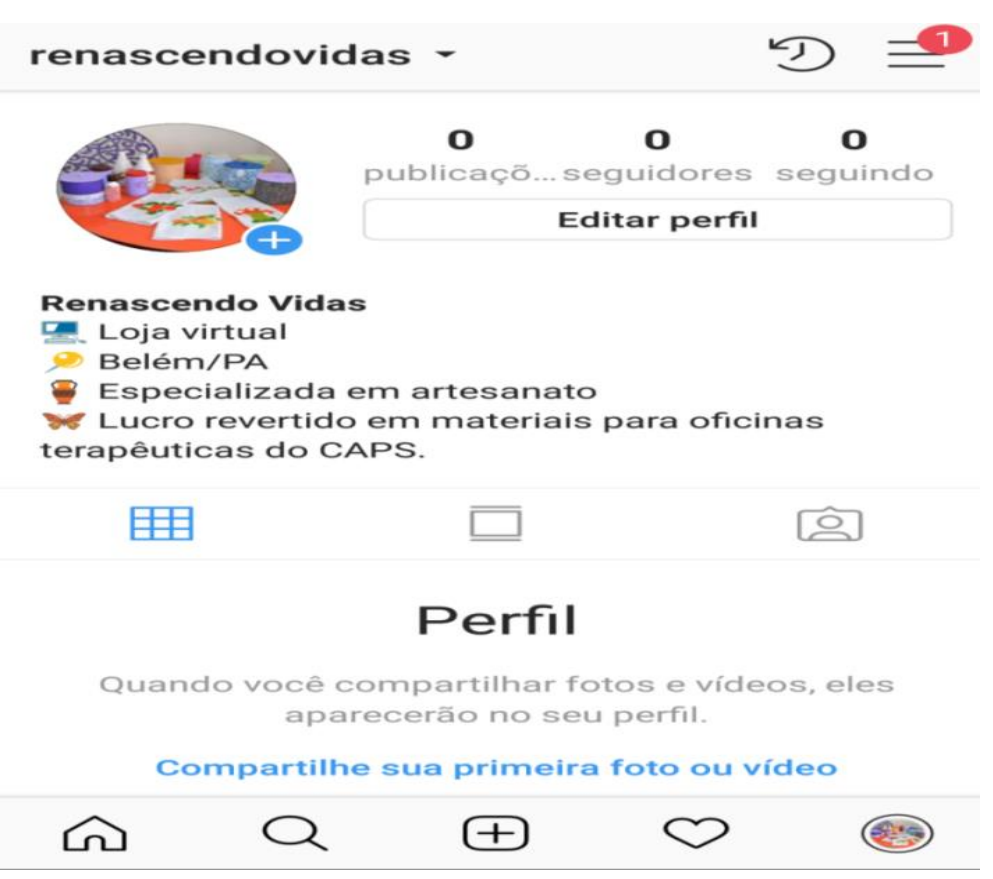

Fonte: Autoria de PENHA JFA, 2019.

Realizando um apanhado histórico, o empreendedorismo na enfermagem é aplicado desde a Guerra da Criméia por Mary Seacole, quando buscou formas de financiar a construção do Hotel Britânico para abrigar os feridos da guerra, desenvolvendo uma loja no local para vender alguns produtos, a fim de conseguir recursos para a manutenção do cuidado (GOUVEIA IM e PINTO JP, 2013).

$\mathrm{Na}$ atualidade um dos recursos bastante utilizados para o desenvolvimento de negócios comerciais são as mídias digitais, que tem como exemplo as lojas virtuais criadas em redes sociais como Instagram e Facebook. Segundo a Associação Brasileira de Comércio Eletrônico, tal modalidade de comércio continuará crescendo nos próximos anos, destacando-se como maior fonte de lucro do mercado (92,64\%) (JÚNIOR VEM, 2018).

Desse modo, verifica-se que a criação de uma loja virtual é uma proposta viável e amplamente utilizada e que pode ser aplicada no CAPS, como forma de obter recursos financeiros para a compra de materiais necessários para as oficinas terapêuticas, como forma de assegurar o cuidado.

\section{CONSIDERAÇÕES FINAIS}

As oficinas terapêuticas são essenciais para os usuários do CAPS, portanto a criação de uma loja virtual, a fim de divulgar os trabalhos realizados pelos usuários durante essas oficinas é uma alternativa viável para a obtenção de renda. Além disso, com a implantação do empreendedorismo no CAPS, através da utilização de mídias sociais na venda, pode-se incentivar não só a execução das oficinas terapêuticas, mas também a divulgação dos trabalhos desenvolvidos e da importância do funcionamento deste serviço de saúde mental, propiciando visibilidade para todo o cuidado prestado e o quão o mesmo é imprescindível para a saúde mental dos usuários.

\section{REFERÊNCIAS}

1. ANDRADE AC, BEN LWD, SANNA MC et al. Empreendedorismo na Enfermagem: panorama das empresas no Estado de São Paulo. Revista Brasileira de Enfermagem, 2015; 1(68): 40-44.

2. BERBEL NAN. As metodologias ativas e a promoção da autonomia de estudantes. Semina: Ciências Sociais e Humanas, 2011; 32(1), 25-40.

3. BRASIL. Ministério da Saúde. Centro de Atenção Psicossocial (CAPS), Brasília: Ministério da Saúde; 2017. 
4. BRASIL. Ministério da Saúde. Saúde mental no SUS: os centros de atenção psicossocial, Brasília: Ministério da Saúde; 2004. 5. BRASIL. Ministério da saúde. Centro de Atenção Psicossocial (CAPS), 2017. Disponível em: http://portalms.saude.gov.br/noticias/693-acoes-e-programas/41146-centro-de-atencao-psicossocial-caps. Acesso em: 28 mai. 2019. 6. BRASIL. Ministério da Saúde. Portaria $n \cong 3.588$, de 21 de dezembro de 2017 , altera as portarias de consolidação $n^{\circ} 3$ e $n^{\circ} 6$, de 28 de setembro de 2017, para dispor sobre a rede de Atenção Psicossocial, e dá outras providencias. Diário Oficial da União. Brasília, 21 de dezembro de 2017. Disponível em: http://www.bvsms.saude.gov.br/. Acesso em: 28 mai. 2019.

7. GANDOLFI FA, RONDINA JM, RONDINA AMF, SOLER ZASG et al. Utilização de tecnologias interativas da Web para orientação de projetos de iniciação científica na área da saúde. Enfermagem Brasileira. 2017; 16(4): 216-225.

8. GOVEIA IM, PINTO JP. Empreendedorismo na Enfermagem Brasileira: Revisão de Literatura. In: Anais do Congresso Nacional de Iniciação Científica, 2013; São Paulo. São Paulo: CONIC-SEMESP; 2013.

9. IBIAPINA ARS, et al. Oficinas terapêuticas e as mudanças em pacientes com transtorno mental. Escola Anna Nery Revista de Enfermagem, 2017; 21(3), 1-8.

10. JÚNIOR VEM. Ideias de Negócios: como montar uma loja virtual - Empreendedorismo.1. ed. Brasília: SEBRAE editora; [2018?].

11. MASON C, BARRAKET J, FRIEL S, O'ROURKE K, STENTA CP. Social innovation for the promotion of health equity. Health Promotion Internships Jobs, 2015; 30(2): 116-125.

12. NUNES BS, SCHREIBER D, SCHMIDT S, MONTARDO SO. Redes Estratégicas Mediadas por Plataformas Digitais na Indústria Criativa: Proposta de um Quadro Teórico. Revista Gestão e Planejamento [internet]. 2018; 19(1): 177-190.

13. PEREIRA JA, BERNARDO A. Empreendedorismo Digital: Estudo do Projeto Negócios Digitais Realizado pelo Sebrae - PR em Maringá. Desenvolvimento em Questão. Revista Contexto e Saúde, 2016; 14(37): 293-327.

14. PITTA A. O que é reabilitação psicossocial no Brasil hoje? In: PITTA, A. Reabilitação psicossocial no Brasil. São Paulo: HUCITEC, 1996. 19-26p.

15. RIBEIRO LA.; SALA, ALB; OLIVEIRA, AGB. As oficinas terapêuticas nos centros de atenção psicossocial. Revista Mineira de Enfermagem[online], 2008, 12(4).

16. TENÓRIO F. A reforma psiquiátrica brasileira, da década de 1980 aos dias atuais: história e conceito. História, Ciências, Saú de Manguinhos, 2002; 9, (1), 25-59. 